\title{
Computer Experiments with Mersenne Primes
}

\author{
M. Wolf \\ Cardinal Stefan Wyszynski University \\ Faculty of Mathematics and Natural Sciences, College of Sciences \\ ul. Wóycickiego 1/3, Auditorium Maximum, (room 113) \\ PL-01-938 Warsaw, Poland \\ E-mail: m.wolf@uksw.edu.pl
}

Received: 14 March 2013; revised: 1 July 2013; accepted: 17 July 2013; published online: 5 September 2013

\begin{abstract}
We have calculated on the computer the sum $\overline{\mathcal{B}}_{M}$ of reciprocals of first 47 known Mersenne primes with the accuracy of over 12000000 decimal digits. Next we developed $\overline{\mathcal{B}}_{M}$ into the continued fraction and calculated geometrical means of the partial denominators of the continued fraction expansion of $\overline{\mathcal{B}}_{M}$. We get values converging to the Khinchin's constant. Next we calculated the $n$-th square roots of the denominators of the $n$-th convergents of these continued fractions obtaining values approaching the Khinchin-Lèvy constant. These two results suggests that the sum of reciprocals of all Mersenne primes is irrational, supporting the common belief that there is an infinity of the Mersenne primes. For comparison we have done the same procedures with a slightly modified set of 47 numbers obtaining quite different results. Next we investigated the continued fraction whose partial quotients are Mersenne primes and we argue that it should be transcendental.
\end{abstract}

Key words: Mersenne primes, continued fractions, Khinchin's constant

\section{INTRODUCTION}

The Mersenne primes $\mathcal{M}_{n}$ are primes of the form $2^{p}-1$ where $p$ must be a prime, see e.g. [18, Sect. 2.VII]. The set of Mersenne primes starts with $\mathcal{M}_{1}=2^{2}-1, \mathcal{M}_{2}=2^{3}-$ $1, \mathcal{M}_{3}=2^{5}-1$ and only 48 primes of this form are currently known, see Great Internet Mersenne Prime Search (GIMPS) at www.mersenne.org. The largest known Mersenne prime has the value $\mathcal{M}_{48}=2^{57885161}-1=5.819 \ldots \times 10^{17425169}$. In general the largest known primes are the Mersenne primes, as the Lucas-Lehmer primality test applicable only to numbers of the form $2^{p}-1$ needs a small multiple of $p$ steps, thus the complexity of checking primality of $\mathcal{M}_{n}$ is $\mathcal{O}\left(\log \left(\mathcal{M}_{n}\right)\right)$. Let us remark that algorithm of Agrawal, Kayal and Saxena (AKS) for arbitrary prime $p$ works in about $\mathcal{O}\left(\log ^{7.5}(p)\right)$ steps and modification by Lenstra and Pomerance has complexity $\mathcal{O}\left(\log ^{6}(p)\right)$.

There is no proof of the infinitude of $\mathcal{M}_{n}$, but a common belief is that as there are presumably infinitely many even perfect numbers thus there is also an infinity of Mersenne primes, see e.g. [9, sect. 16.8]. S. S. Wagstaff Jr. in [26] (see also $[21, \S 3.5]$ ) gave heuristic arguments that $\mathcal{M}_{n}$ grow doubly exponentially:

$$
\log _{2} \log _{2} \mathcal{M}_{n} \sim n e^{-\gamma}
$$

where $\gamma=0.57721566 \ldots$ is the Euler-Mascheroni constant. In Fig. 1 we compare the Wagstaff conjecture with all 48 presently known Mersenne primes $\mathcal{M}_{n}$. Of these 48 known $\mathcal{M}_{n}=2^{p}-1$ there are 28 with $p \bmod 4=1$ and 19 with $p \bmod 4=3$. It is in opposite to the set of all primes where the phenomenon of Chebyshev bias is known: for initial primes there are more primes $p \equiv 3(\bmod 4)$ than $p \equiv 1$ $(\bmod 4),[11,19]$.

In this paper we are going to exploit two facts about the continued fractions to support the conjecture on the infinitude of Mersenne primes: the existence of the Khinchin constant and Khinchin-Lèvy constant. We calculate the sum of reciprocals of the Mersenne primes $\mathcal{B}_{M}=\sum_{n} 1 / \mathcal{M}_{n}$; if there is infinity of Mersenne primes then this number $\mathcal{B}_{M}$ should be irrational (at least, because it is probably even transcendental, as it is difficult to imagine the polynomial with some mysterious integer coefficients whose one of roots should be $\mathcal{B}_{M}$ ). 


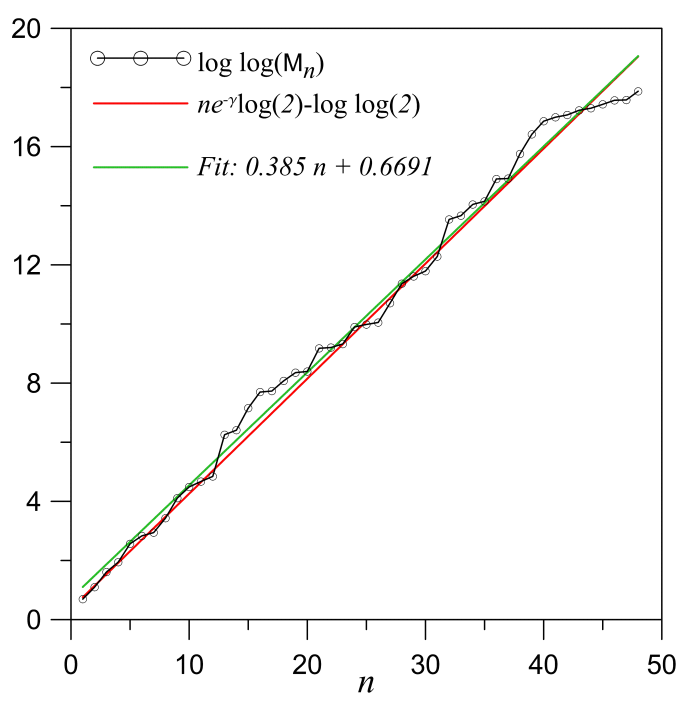

Fig. 1. The plot of $\log \log \left(\mathcal{M}_{n}\right)$ and the Wagstaff conjecture (1). The fit was made to all known $\mathcal{M}_{n}$ and it is $0.382 n+0.7229$, while $n e^{-\gamma} \log (2)-\log \log (2) \approx 0.3892 n+0.3665$. The rather good coincidence of $\log \log \left(\mathcal{M}_{n}\right)$ and (1) is seeming, as to get original $\mathcal{M}_{n}$ 's the errors are amplified to huge values by double exponentiation

There exists a method based on the continued fraction expansion which allows to detect whether a given number $r$ can be irrational or not. Let

$$
\begin{aligned}
& r=\left[a_{0}(r) ; a_{1}(r), a_{2}(r), a_{3}(r), \ldots\right] \equiv \\
& \equiv a_{0}(r)+\frac{1}{a_{1}(r)+\frac{1}{a_{2}(r)+\frac{1}{a_{3}(r)+\ddots}}}
\end{aligned}
$$

be the continued fraction expansion of the real number $r$, where $a_{0}(r)$ is an integer and all $a_{k}(r)$ with $k \geq 1$ are positive integers. The quantities $a_{k}(r)$ are called partial quotients or partial denominators. Khinchin has proved [13], see also [20], that

$$
\begin{aligned}
& \lim _{n \rightarrow \infty}\left(a_{1}(r) \ldots a_{n}(r)\right)^{\frac{1}{n}}= \\
&= \prod_{m=1}^{\infty}\left\{1+\frac{1}{m(m+2)}\right\}^{\log _{2} m} \\
& \equiv K \approx 2.685452001
\end{aligned}
$$

is a constant for almost all real $r[8, \S 1.8]$ (the term $a_{0}$ is skipped in (3)). The exceptions are of the Lebesgue measure zero and include rational numbers, quadratic irrationals and some irrational numbers too, like for example the Euler constant $e=\lim _{n \rightarrow \infty}\left(1+\frac{1}{n}\right)^{n}=2.7182818285 \ldots$ for which the $n$-th geometrical mean tends to infinity like $\sqrt[3]{n}$, see $[10, \S 14.3$ (p.160)]. The constant $K$ is called the Khinchin constant. If the sequence

$$
K(r ; n)=\left(a_{1}(r) a_{2}(r) \ldots a_{n}(r)\right)^{\frac{1}{n}}
$$

for a given number $r$ tend to $K$ for $n \rightarrow \infty$ we can regard it as an indication that $r$ is irrational - all rational numbers have a finite number of partial quotients in the continued fraction expansion and hence starting with some $n_{0}$ will be $a_{n}=0$ for all $n>n_{0}$. It is possible to construct a sequence of rational numbers such that the geometrical means of partial quotients of their continued fraction will tend to the Khinchin constant (A. Schinzel, private communication).

The Khinchin-Lèvy's constant arises in the following way: Let the rational $P_{n}(r) / Q_{n}(r)$ be the $n$-th partial convergent of the continued fraction of $r$ :

$$
\frac{P_{n}(r)}{Q_{n}(r)}=\left[a_{0}(r) ; a_{1}(r), a_{2}(r), a_{3}(r), \ldots, a_{n}(r)\right] .
$$

In 1935 Khinchin [12] has proved that for almost all real numbers $r$ the denominators $Q_{n}$ of the convergents fulfill:

$$
\lim _{n \rightarrow \infty}\left(Q_{n}(r)\right)^{1 / n} \equiv \lim _{n \rightarrow \infty} L(r ; n)=L
$$

and in 1936 Paul Levy [14] found an explicit expression for this constant $L$ :

$$
\lim _{n \rightarrow \infty} \sqrt[n]{Q_{n}(r)}=e^{\pi^{2} / 12 \log (2)} \equiv L=3.27582291872 \ldots
$$

$L$ is called the Khinchin-Lèvy's constant $[8, \S 1.8]$. Again the set of exceptions to the above limit is of the Lebesgue measure zero and it includes rational numbers, quadratic irrationals etc.

We have made all computer calculations for first 47 Mersenne primes, i.e. up to $\mathcal{M}_{47}=2^{43112609}-1=$ $3.1647026933 \ldots \times 10^{12978188}$. Because the new (it is not known whether there exist undiscovered primes of the form $2^{p}-1$ between $\mathcal{M}_{42}$ and the largest presently known Mersenne prime) Mersenne prime discovered in January 2013 is about five millions orders larger than $\mathcal{M}_{47}$ to include it in the computer experiments would require a few additional months of CPU time.

\section{FIRST EXPERIMENT}

Let us define the sum of reciprocals of all Mersenne primes:

$$
\mathcal{B}_{M}=\sum_{n=1}^{\infty} \frac{1}{\mathcal{M}_{n}}
$$

which can be regarded as the analog of the Brun's constant, i.e. the sum of reciprocals of all twin primes:

$$
\mathcal{B}_{2}=\left(\frac{1}{3}+\frac{1}{5}\right)+\left(\frac{1}{5}+\frac{1}{7}\right)+\left(\frac{1}{11}+\frac{1}{13}\right)+\ldots
$$

In 1919 Brun [5] has shown that this constant $\mathcal{B}_{2}$ is finite, thus leaving the problem of infinity of twin primes not 
decided. Today's best numerical value is $\mathcal{B}_{2} \approx 1.90216058$, see [16], [22]. Yet it is possible to prove that there is infinity of twins by showing that Brun's constant is irrational [27] (we believe it is even transcendental).

Using PARI [24] we have calculated the sum of reciprocals of all known 47 Mersenne primes $\overline{\mathcal{B}}_{M}$ with accuracy over 12 million digits:

$$
\begin{array}{r}
\overline{\mathcal{B}}_{M}=0 \\
0.5164541789407885653304873429 \\
715228588159685534154197 \ldots
\end{array}
$$

This number is not recognized by the Symbolic Inverse Calculator (http://pi.lacim.uqam.ca) maintained by Simone Plouffe. The bar over $\mathcal{B}_{M}$ denotes the finite (at present consisting of 47 terms) approximation to the full sum defined in (8). It is not known whether there are Mersenne prime numbers with exponent $25964951<p<43112609$-currently confirmed by GIMPS is that $2^{25964951}-1$ is the 42-th Mersenne prime $\mathcal{M}_{42}-$ it is not known whether any undiscovered Mersenne primes exist between the 42th $\mathcal{M}_{42}$ and the 42th Mersenne prime $\mathcal{M}_{48}$. We have taken 12000035 digits of $\overline{\mathcal{B}}_{M}$ - it means that we assume that there are no unknown Mersenne primes with $p<12000035 \log (10) / \log (2) \approx$ 39863253 (as for June 2013 GIMPS has checked all exponents below 44457869 at least once). Using the incredibly fast procedure ContinuedFraction $[\cdot]$ implemented in Mathematica ${ }^{\complement}$ we calculated the continued fraction expansion of $\overline{\mathcal{B}}_{M}$. The result was built from 11645012 partial denominators $a_{1}=1, a_{2}=1, a_{3}=14, \ldots, a_{11645012}=4$. The $n$-th convergent $P_{n}(r) / Q_{n}(r)$, see (5), fulfills

$$
\left|r-\frac{P_{k}}{Q_{k}}\right|<\frac{1}{Q_{k} Q_{k+1}}<\frac{1}{Q_{k}^{2} a_{k+1}} \leq \frac{1}{Q_{k}^{2}},
$$

see [13, Theorem 9, p.9]. From this it follows that if $r$ is known with precision of $d$ decimal digits we can continue calculation of quotients $a_{n}$ up to such $n$ that the denominator of the $n$-th convergent $Q_{n}^{2}<10^{d}$. We have checked that $Q_{11645012}=4.291385 \times 10^{6000016}$ and apparently $Q_{11645013}^{2}>10^{12000035}$.

The largest partial quotient was $a_{9965536}=716699617$. We have checked correctness of the continued fraction expansion of $\overline{\mathcal{B}}_{M}$ by calculating backwards from $[0 ; 1,1,14, \ldots, 4]$ the partial convergent. The Mathematica ${ }^{\complement}$ has built in the procedure FromContinuedFraction $[\cdot]$, but we have used our own procedure written in PARI and implementing the recurrence:

$$
P_{n+1}=a_{n} P_{n}+P_{n-1}, \quad Q_{n+1}=a_{n} Q_{n}+Q_{n-1}, \quad n \geq 1
$$

with initial values

$$
P_{0}=a_{0}, \quad Q_{0}=1, \quad P_{1}=a_{0} a_{1}+1, \quad Q_{1}=a_{1} .
$$

We have obtained the ratio of two coprime 6000018 decimal digits long integers (it means denominator was of the order $10^{6000018}$ and hence its square was smaller than $10^{12000035}$, see eq.(11)):

6000018 digits

$$
\frac{2216304109121123313251143869 \ldots 2210}{4291385759849224534616716035 \ldots 2813}
$$

whose ratio has 12000033 digits the same as $\overline{\mathcal{B}}_{M}$. The decimal expansion of $\overline{\mathcal{B}}_{M}=P / Q$ is obviously periodic (recurring), see [9, Th. 135], but the length of the period is much larger than $10^{12000000}$. According to the Theorem 135 from [9] the period $r$ of the decimal expansion of $\overline{\mathcal{B}}_{M}$ is equal to the order of $10 \bmod Q$, i.e. it is the smallest positive $r$ for which

$$
10^{r} \equiv 1(\bmod Q)
$$

Because $Q$ being the product of all 47 Mersenne primes is of the order $3.509 \ldots \times 10^{86789810}$, we expect that the value of $r$ is much larger than $10^{12000000}$.

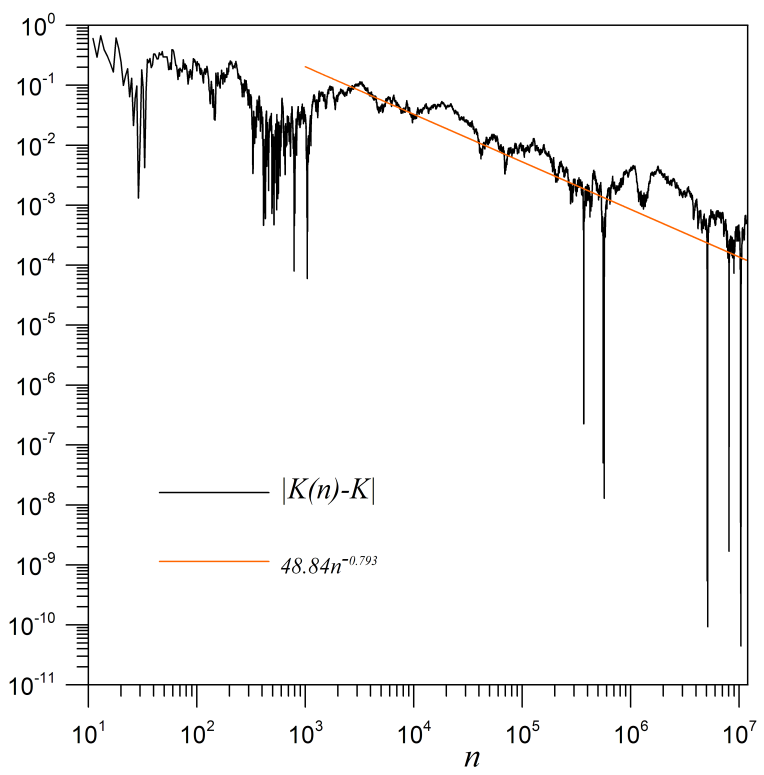

Fig. 2. The plot showing the distance to $K$ of the running geometrical averages $K(n)=\left(a_{1} a_{2} \cdots a_{n}\right)^{1 / n}$ for the continued fraction of $\mathcal{B}_{M}$

From the sequence of partial quotients $a_{1}=1, a_{2}=$ $1, a_{3}=14, \ldots, a_{11645012}=4$ we have calculated running geometrical means

$$
K(n)=\left(\prod_{k=1}^{n} a_{k}\right)^{1 / n}
$$


for $n=11, \ldots, 11645012$. The obtained numbers $K(n)$ quickly tend to the Khinchine constant thus in Fig. 2 we have plotted the differences $|K(n)-K|$. The power fit to the values for $n=1000 \ldots 11645012$ gives the decrease of the form $|K(n)-K| \sim n^{-0.79}$ and it suggests that indeed $\lim _{n \rightarrow \infty} K(n)=K$ and thus $\mathcal{B}_{M}$ is irrational. Indices $n$ for which the geometric means $K(n)$ produce progressively better approximations to Khinchin's constant are:

$$
\begin{aligned}
& 1,3,12,16,17,21,24,26,29,412,788,1045, \\
& 369625,369636, \ldots, 5137093,10389989
\end{aligned}
$$

the smallest value of $|K(n)-K|$ was $4.455957 \ldots \times 10^{-11}$. This sequence can be regarded as the counterpart to the A048613 at OEIS.org.

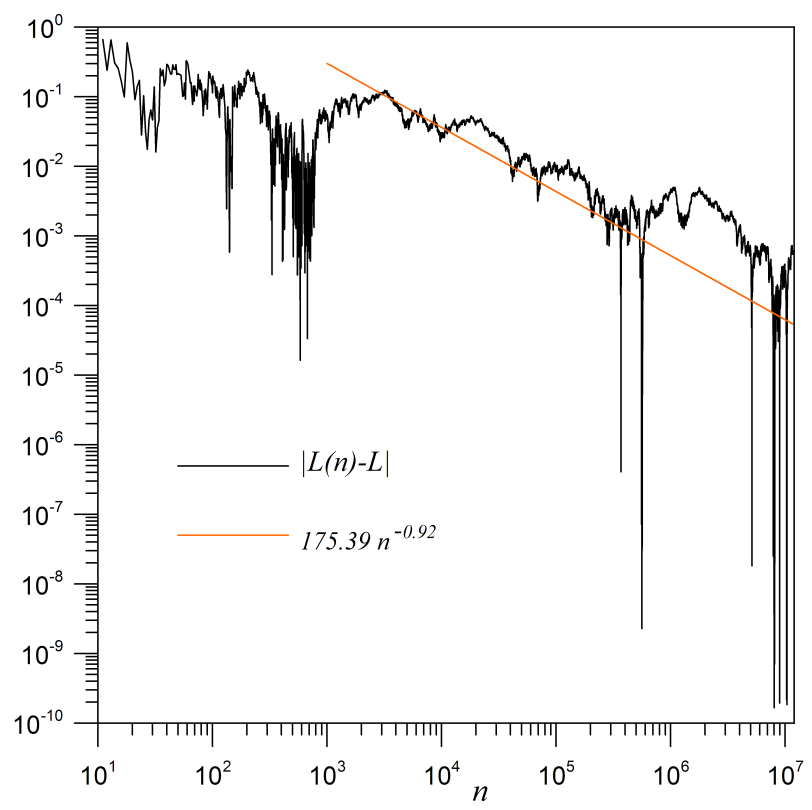

Fig. 3. The plot showing the distance to $L$ of the $(Q(n))^{1 / n}$ obtained from the partial convergents of the continued fraction of $\mathcal{B}_{M}$ for $n=11, \ldots, 11645012$

Next we calculated running (i.e. for $n=11, \ldots$, 11645012) partial quotients $P_{n} / Q_{n}$ and then the quantities $L(n)=\sqrt[n]{Q_{n}}$, which for almost all irrational numbers should tend to the Khinchine-Levy constant. The behaviour of $\sqrt[n]{Q_{n}}$ is shown in Fig. 3. Again we see that these quantities tend to the limit $L$; the fitting of the power-like dependence for $n>10$ gives that $|L(n)-L| \approx 175.39 n^{-0.92}$. The shape of the plot in this figure is similar to the plot of $|K(n)-K|$ in Fig. 2.

Both differences $K(n)-K$ and $L(n)-L$ have a lot of sign changes for $n<11645012$. Figures 4 and 5 present the plots of these differences together with the number of sign changes.

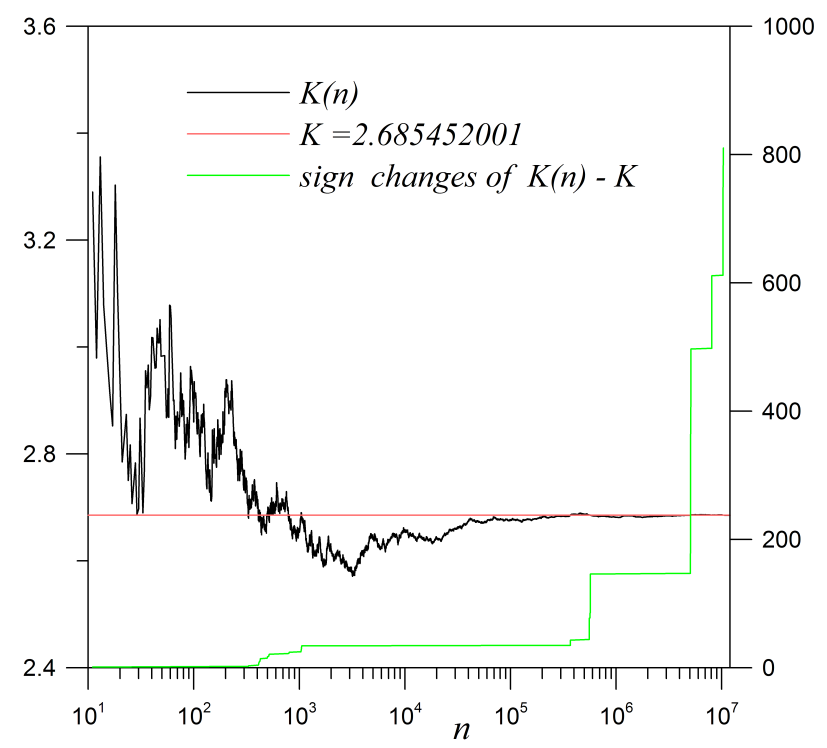

Fig. 4. The plot of $K(n)$ in black approaching the Khinchine constant $K=2.685452 \ldots$. (in red) with values presented on left $y$-axis. In green are presented numbers of sign changes of $K(n)-K$ up to $n$-the right $y$-axis is for this plot

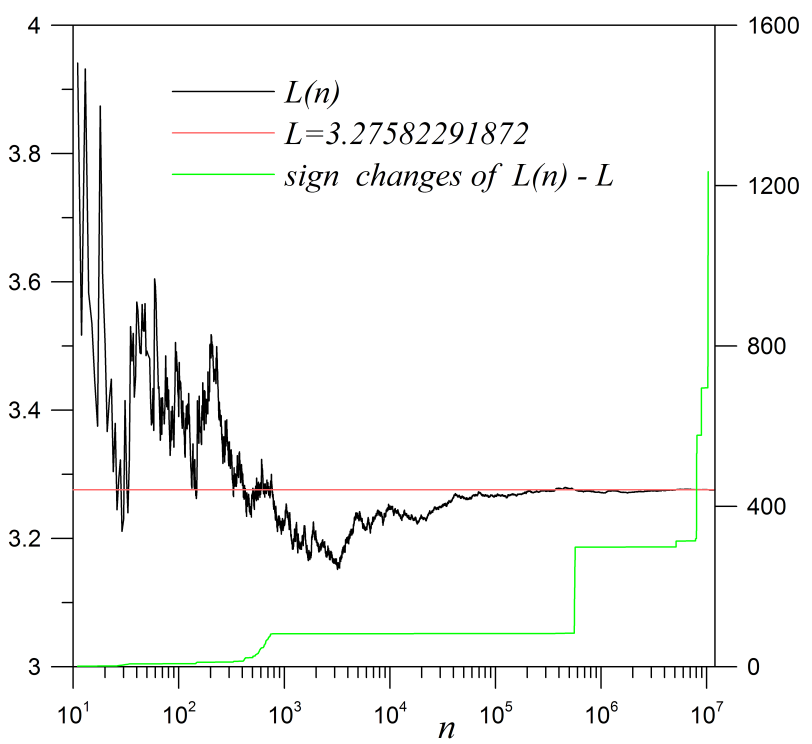

Fig. 5. The plot of $L(n)$ in black approaching the Khinchine-Levy constant $L=3.27582291872 \ldots$ (in red) with values presented on left $y$-axis. In green are presented numbers of sign changes of $L(n)-L$ up to $n$ - the right $y$-axis is for this plot

The data presented in Figures 2 and 3 provide the hints that $\mathcal{B}_{M}$ is irrational and hence that there is infinity of Mersenne primes. But we are convinced $\mathcal{B}_{M}$ is in fact transcendental. In favor of this claim we recall here the result of A. J. van der Poorten and J. Shallit [25] that the following sum 


$$
\frac{1}{2^{1}}+\frac{1}{2^{2}}+\frac{1}{2^{3}}+\frac{1}{2^{5}}+\ldots+\frac{1}{2^{F_{n}}}+\ldots
$$

where $F_{n}$ are Fibonacci numbers, is transcendental. It is well known that the Liouville number

$$
\frac{1}{2^{1 !}}+\frac{1}{2^{2 !}}+\frac{1}{2^{3 !}}+\frac{1}{2^{4 !}}+\cdots+\frac{1}{2^{n !}}+\ldots
$$

is transcendental, see [9, Theorem 192]. In $\mathcal{B}_{M}$, assuming the Wagstaff conjecture, unfortunately the terms decrease slower: $n !>2^{n}>2^{e^{-\gamma} n}$ for $n \geq 4$ but faster than $F_{n}=\left\lfloor\frac{\varphi^{n}}{\sqrt{5}}+\frac{1}{2}\right\rfloor$, where $\varphi=\frac{1+\sqrt{5}}{2} \approx 1.6180339887 \ldots$

Let $\psi_{n}(m)$ denote the number of partial quotients $a_{k}$ with $k=1,2, \ldots, n$ which are equal to $m$ :

$$
\psi_{n}(m)=\sharp\left\{k: k \leq n \text { and } a_{k}=m\right\} .
$$

Then the Gauss-Kuzmin theorem (for excellent exposition see e.g. $[10, \S 14.3])$ asserts that

$$
\lim _{n \rightarrow \infty} \frac{\psi_{n}(m)}{n}=\frac{\log \left(1+\frac{1}{m(m+2)}\right)}{\log (2)}
$$

for continued fractions of almost all real numbers. In other words, the probability to find the partial quotient $a_{k}=m$ is equal to $\log _{2}(1+1 / m(m+2))$. In Fig. 6 we present the plot of the $\frac{\psi_{11645013}(\mathrm{~m})}{11645013}$ for the continued fraction of $\overline{\mathcal{B}}_{M}$ and $m=1,2, \ldots 1000$ together with prediction given by the Gauss-Kuzmin theorem finding excellent agreement.

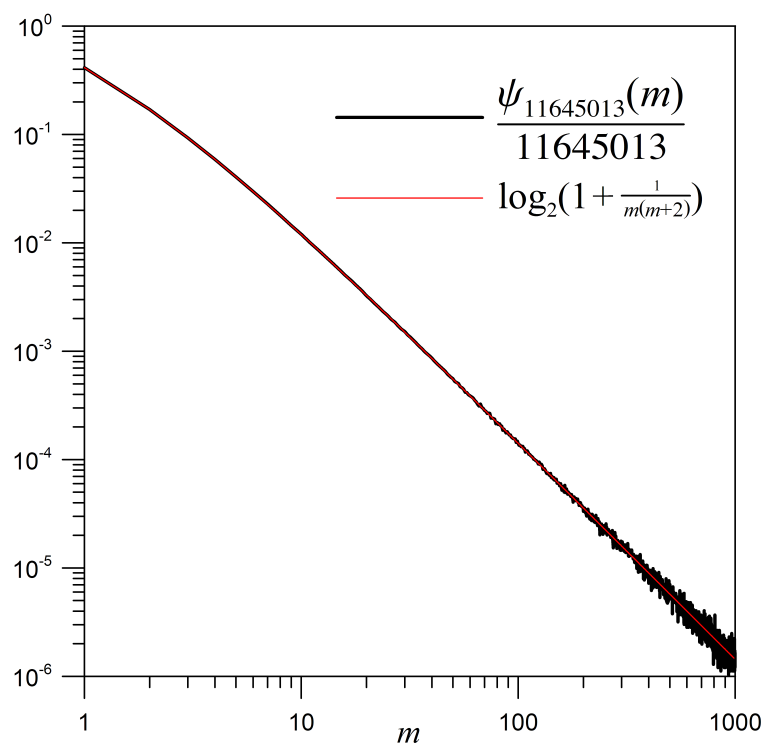

Fig. 6. The plot of the measured for the continued fraction of $\mathcal{B}_{M}$ probability to find the partial quotient $a_{k}=m$ for the continued fraction of $\mathcal{B}_{M}$
Finally let us notice that the number $\overline{\mathcal{B}}_{M}$ computed with 12000035 digits is normal in the base 10, see Table 1.

Tab. 1. Illustration of the normality of $\overline{\mathcal{B}}_{M}$ : the numbers in the second (fifth) row give the number of digits $0,1, \ldots 9$ appearing in the decimal expansion of $\overline{\mathcal{B}}_{M}$ and the third (sixth) rows contain the ratio of numbers in the second row divided by 12000035

\begin{tabular}{c|c|c|c|c}
\hline 0 & 1 & 2 & 3 & 4 \\
\hline 1200553 & 1199322 & 1199420 & 1200548 & 1199397 \\
\hline 0.1000458 & 0.0999432 & 0.0999514 & 0.1000454 & 0.0999495 \\
\hline \hline 5 & 6 & 7 & 8 & 9 \\
\hline 1198596 & 1200876 & 1200056 & 1201757 & 1199510 \\
\hline 0.0998827 & 0.1000727 & 0.1000044 & 0.1001461 & 0.0999589 \\
\hline
\end{tabular}

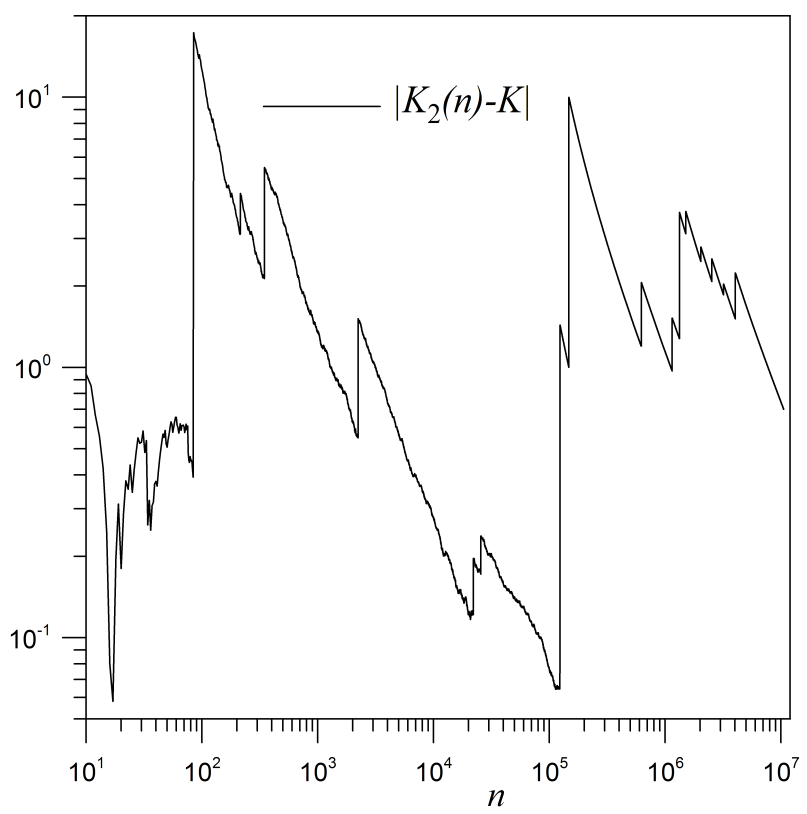

Fig. 7. The plot showing the distance to $K$ of the running geometrical averages $K_{2}(n)$ for the continued fraction of $\mathcal{S}$ for $n=11, \ldots, 10550114$

For comparison we have repeated the above procedure for an artificial set of 47 numbers of the size corresponding to the known Mersenne primes. We have simply skipped -1 in the Mersenne primes and using PARI we have computed over 120000000 digits of the sum:

$$
\mathcal{S}=\frac{1}{2^{2}}+\frac{1}{2^{3}}+\ldots+\frac{1}{2^{42643801}}+\frac{1}{2^{43112609}}
$$

This number $\mathcal{S}$ is the ratio of the form $A / 2^{43112609}$, where $\operatorname{gcd}(A, 243112609)=1$. From $[9, \S 9.2]$ we know that $\mathcal{S}$ has terminating decimal expansion consisting of 43112609 
decimal digits, thus calculating 12000000 digits of this sum makes sens as it does not contain recurring periodic patterns of digits. We have developed $\mathcal{S}$ into the continued fraction, which resulted in 10550114 partial quotients. The calculated quantities for this case we denote with the subscript 2 : $Q_{2}(n), K_{2}(n), L_{2}(n)$ to distinguish them from earlier experiment for $\overline{\mathcal{B}}_{M}$. We have calculated the running geometrical averages of the partial quotients $K_{2}(n)$ and the results are presented in Figure 7. Next we calculated partial convergents $P_{2}(n) / Q_{2}(n), n=1,2, \ldots, 10550114$ and from them the quantities $L_{2}(n) \equiv\left(Q_{2}(n)\right)^{1 / n}$, which should tend to the Levy constant $L$. In Figure 8 the differences $\left|L_{2}(n)-L\right|$ are plotted. Obtained plots are completely different from those seen in Figures 2 and 3 and they suggest $K_{2}(n)$ as well as $L_{2}(n)$ for number $\mathcal{S}$ do not have the limit. In this artificial case we have encountered the phenomenon of extremely large partial denominators: there were $a_{n}$ of the order $10^{70548}, 10^{97732}$ and $10^{279910}$. These large partial denominators are responsible for the smaller number of $a_{k}$ than for $\overline{\mathcal{B}}_{M}$, see (11).

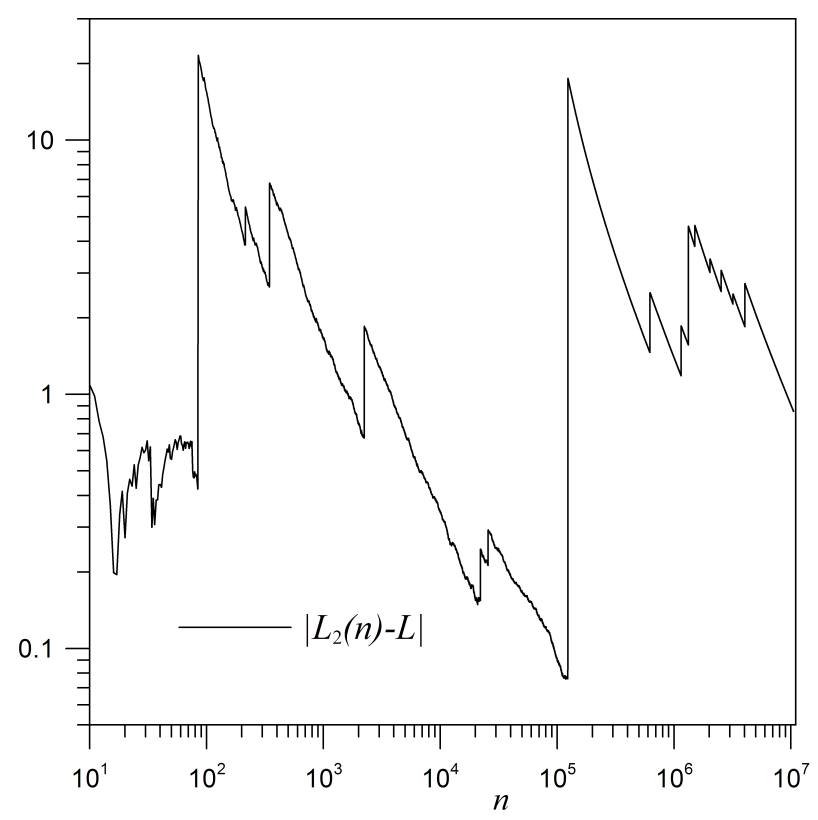

Fig. 8. The plot showing the distance to $L$ of the $\left(Q_{2}(n)\right)^{1 / n}$ obtained from the partial convergents of the continued fraction of $\mathcal{S}$ for $n=11, \ldots, 10550114$

\section{SECOND EXPERIMENT}

Let us define the supposedly infinite and convergent continued fraction $u_{\mathcal{M}}$ by taking $a_{n}=\mathcal{M}_{n}$ :

$$
\begin{aligned}
u_{\mathcal{M}}= & {[0 ; 3,7,31,127,8191,} \\
& 131071,524287,2147483647, \ldots]
\end{aligned}
$$

Using 33 Mersenne primes $3,7,31, \ldots, 2^{859433}-1$ in a couple of minutes we have calculated $u_{\mathcal{M}}$ with the precision of 10000000 digits; first 50 digits of $u_{\mathcal{M}}$ are:

$$
\begin{array}{r}
u_{\mathcal{M}}=0.318248158405844869425962 \\
02748140694243806236564 \ldots
\end{array}
$$

This number is not recognized by the Symbolic Inverse Calculator (http://pi.lacim.uqam.ca). Because $1 / Q_{47}^{2}\left(u_{\mathcal{M}}\right) \approx$ $1.84313 \times 10^{-173579621}$ it follows from (11) that theoretically it is possible to obtain the value of $u_{\mathcal{M}}$ from presently known 47 Mersenne primes with over 170,000,000 decimal digits of accuracy. Obviously $u_{\mathcal{M}}$ is the exception to the Khinchin and Levy Theorems in view of the very fast growth of $\mathcal{M}_{n}$ - see the Wagstaff [26] conjecture (1). We will present below conditional arguments that $u_{\mathcal{M}}$ is transcendental.

There is vast literature concerning the transcendentality of continued fractions. For example, the continued fraction

$$
\left[0 ; 2^{1 !}, 2^{2 !}, 2^{3 !}, \ldots, 2^{n !}, \ldots\right]
$$

is transcendental, see [9, Theorem 192], [23, Example 4].

The Theorem of H. Davenport and K.F. Roth [7] states that if the denominators $Q_{n}$ of convergents of the continued fraction $r=\left[a_{0} ; a_{1}, a_{2}, \ldots\right]$ fulfill

$$
\limsup _{n} \frac{\sqrt{\log (n)} \log \left(\log \left(Q_{n}(r)\right)\right)}{n}=\infty
$$

then $r$ is transcendental. This theorem requires for the transcendence of $r$ very fast increase of denominators of the convergents: at least doubly exponential growth is required for (23). The set of continued fractions which can satisfy the Theorem of H. Davenport and K.F. Roth is of measure zero, as it follows from the Theorem 31 from the Khinchin's book [13], which asserts there exists an absolute constant $B$ such that for almost all real numbers $r$ and sufficiently large $n$ the denominators of its continued fractions satisfy:

$$
Q_{n}(r)<e^{B n}
$$

The paper of A. Baker [4] from 1962 contains a few theorems on the transcendentality of Maillet type continued fractions [15], i. e. continued fractions with bounded partial quotients which have transcencendental values. Besides Maillet continued fractions there are some specific families of other continued fractions of which it is known that they are transcendental. In the papers $[2,17]$ it was proved that the Thue-Morse continued fractions with bounded partial quotients are transcendental. Quite recently there appeared the preprint [6] where the transcendence of the Rosen continued fractions was established. For more examples see [3]. 


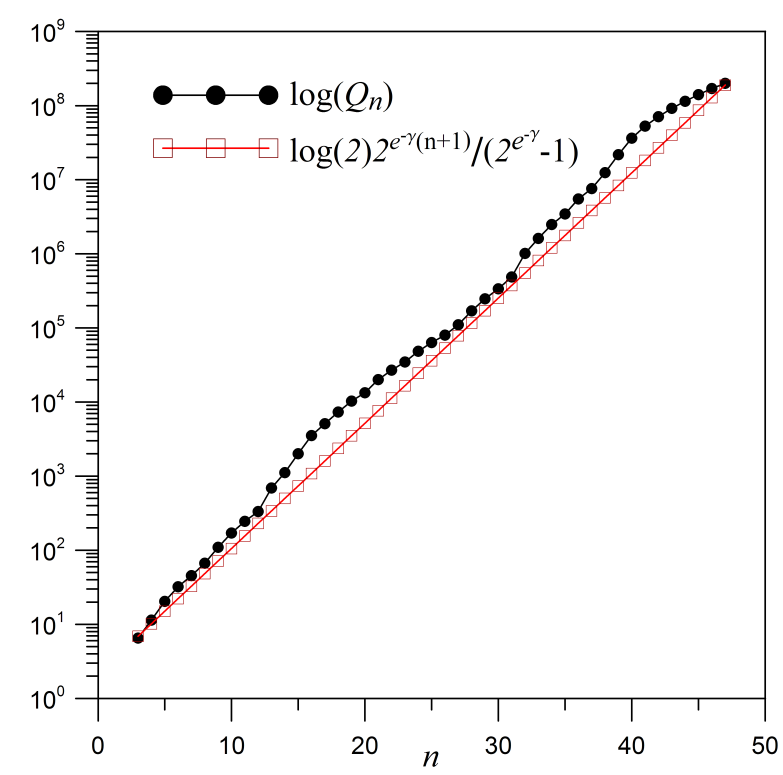

Fig. 9. Illustration of the inequality (26) for $3 \leq n \leq 47$. Although the last points seem to coincide in fact $Q_{47}=2.32928 \ldots \times$ $10^{86789810}$, while $2^{c 2^{48 e^{-\gamma}}}=1.21513 \ldots \times 10^{82034318}$ - hundreds of thousands of orders of difference!

In the paper [1] B. Adamczewski and Y. Bugeaud, among others, have improved (23) to the form: If

$$
\limsup _{n} \frac{\log \left(\log \left(Q_{n}(r)\right)\right)}{n^{2 / 3} \log (n)^{2 / 3} \log (\log (n))}=\infty
$$

then $r$ is transcendental.

Assuming the Wagstaff conjecture $\mathcal{M}_{n} \sim 2^{2^{n e^{-\gamma}}}$ mentioned in the Introduction we obtain that for large $n$

$Q_{n}(\mathcal{M})>2^{c 2^{(n+1) e^{-\gamma}}}, \quad c=\frac{1}{2^{e^{-\gamma}}-1}=2.101893933 \ldots$

and thus the transcendence of $u_{\mathcal{M}}$ will follow from the Davenport-Roth Theorem (23):

$$
\frac{\sqrt{\log (n)} \log \left(\log \left(Q_{n}(\mathcal{M})\right)\right)}{n} \sim \sqrt{\log (n)} \rightarrow \infty .
$$

We illustrate the inequality (26) in Figure 9 - the values of labels on the $y$-axis give an idea of the order of the fast growth of $Q_{n}\left(u_{\mathcal{M}}\right)$ : the largest for $n=47$ is of the order $Q_{47}=e^{1.9984 \ldots \times 10^{8}}=2.32928 \ldots \times 10^{86789810}$, see also Tab. 2.

One of the transcendence criteria is the Thue-Siegel-Roth Theorem, which we recall here in the following form:

Thue-Siegel-Roth Theorem: If there exist such $\epsilon>0$ that for infinitely many fractions $A_{n} / B_{n}$ the inequality

$$
\left|r-\frac{A_{n}}{B_{n}}\right|<\frac{1}{B_{n}^{2+\epsilon}}, \quad n=1,2,3, \ldots,
$$

holds, then $r$ is transcendental.
Tab. 2. A sample of values of inverses of the squares $Q_{n}$ giving

\begin{tabular}{|c|c|}
\hline$n$ & $1 / Q_{n}^{2}$ \\
\hline 3 & $2.131173743 \times 10^{-6}$ \\
\hline 4 & $1.320662319 \times 10^{-10}$ \\
\hline 5 & $1.968416969 \times 10^{-18}$ \\
\hline 6 & $1.145786956 \times 10^{-28}$ \\
\hline 7 & $4.168364565 \times 10^{-40}$ \\
\hline 8 & $9.038699842 \times 10^{-59}$ \\
\hline 9 & $1.699990496 \times 10^{-95}$ \\
\hline$\vdots$ & $\vdots$ \\
\hline 17 & $9.32543401 \times 10^{-4439}$ \\
\hline 18 & $1.38891910 \times 10^{-6375}$ \\
\hline 19 & $3.81534516 \times 10^{-8936}$ \\
\hline 20 & $4.67942175 \times 10^{-11599}$ \\
\hline$\vdots$ & $\vdots$ \\
\hline 40 & $4.50116310 \times 10^{-31553835}$ \\
\hline 41 & $5.02100786 \times 10^{-46025300}$ \\
\hline 42 & $3.36434042 \times 10^{-61657758}$ \\
\hline 43 & $3.38166968 \times 10^{-79961861}$ \\
\hline 44 & $2.17906011 \times 10^{-99578575}$ \\
\hline 45 & $5.32688381 \times 10^{-121949118}$ \\
\hline 46 & $1.84595823 \times 10^{-147623244}$ \\
\hline 47 & $1.84313029 \times 10^{-173579621}$ \\
\hline
\end{tabular}
an idea of the speed of convergence of $\left[0 ; \mathcal{M}_{1}, \mathcal{M}_{2}, \ldots, \mathcal{M}_{n}\right]$ to $u_{\mathcal{M}}$

Let us stress that $\epsilon$ here does not depend on $n-$ it has to be the same for all fractions $A_{n} / B_{n}$. We can test the criterion (28) for $u_{\mathcal{M}}$ using as the rational approximations $A_{n} / B_{n}$ the convergents $P_{n} / Q_{n}$ of the continued fraction (20).

In [23] J. Sondow has given the formula for irrationality exponent of an irrational number $r$ in terms of the continued fraction expansion of $r=\left[a_{0} ; a_{1}, a_{2}, \ldots\right]$ and convergents $P_{n} / Q_{n}$. For our purposes we state his result as the estimation for $\epsilon$ :

$$
\epsilon \leq \limsup _{n \rightarrow \infty} \frac{\log a_{n+1}}{\log Q_{n}} .
$$

Let us denote $\delta \equiv 2+\epsilon$. From the Wagstaff conjecture we obtain that the exponent $\delta$ of $B^{\delta}$ appearing on the r.h.s. of (28) should be of the order

$$
\delta \approx 2+2^{e^{-\gamma}}-1=2.47477 \ldots \quad \text { (i.e. } \epsilon \approx 0.47477 \ldots \text { ) }
$$


implying transcendence of $u_{\mathcal{M}}$. In Fig. 10 we present actual values of $\delta\left(u_{\mathcal{M}} ; n\right)=-\log \left|u_{\mathcal{M}}-P_{n} / Q_{n}\right| / \log \left(Q_{n}\right)$ for $n=3,4, \ldots, 45$ and indeed the values oscillate around $1+2^{e^{-\gamma}}=2.47477 \ldots$

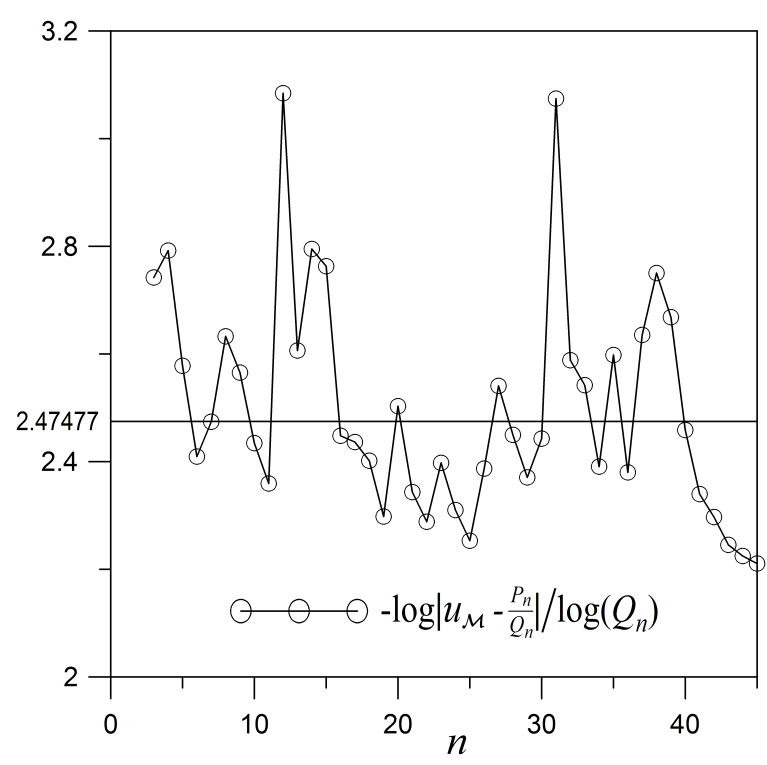

Fig. 10. The plot of $-\log \left|u_{\mathcal{M}}-P_{n} / Q_{n}\right| / \log \left(Q_{n}\right)$ fluctuating around the estimation (30)

First we have calculated $u_{\mathcal{M}}$ using all 47 Mersenne primes with accuracy 140000000 digits and for $n=3,4, \ldots, 45$ we have calculated convergents $P_{n} / Q_{n}$ and next the differences $\left|u_{\mathcal{M}}-P_{n} / Q_{n}\right|$ with accuracy $1 / Q_{n}^{2}$ (see Table 2), from which we determined the $\delta\left(u_{\mathcal{M}} ; n\right)$. The arithmetic average of 43 values $\delta\left(u_{\mathcal{M}} ; n\right)$ is $2.5002 \ldots$, quite close to the estimated value (30). It took a few months of CPU time to collect data presented in Fig. 10: It took 12 days of CPU time on the AMD Opteron $2700 \mathrm{MHz}$ processor to collect data for $n \leq$ 40; the point $n=40$ needed precision of almost 40,000,000 digits, as $\left|u_{\mathcal{M}}-P_{40} / Q_{40}\right|=1.5033 \times 10^{-38789567}$, while $1 / Q_{40}^{2}=4.501 \ldots \times 10^{-31553835}$. To calculate the difference $\left|u_{\mathcal{M}}-P_{n} / Q_{n}\right|$ for $n=41,42,43$ the precision of 100000000 digits was needed. For $n=44$ and $n=45$ the difference $\left|u_{\mathcal{M}}-P_{n} / Q_{n}\right|$ was calculated with the precision 130000000 digits (see Table 2 for $n=44$ and $n=45$ ) and it took about one month of CPU time for each point.

\section{Conclusion}

The performed experiments provide arguments in favour of the common belief that there is an infinity of Mersenne Primes.

\section{Acknowledgment}

I thank Prof. Andrzej Schinzel and Prof. Jonathan Sondow for e-mail correspondence and useful remarks.

\section{References}

[1] B. Adamczewski and Y. Bugeaud, On the Maillet-Baker continued fractions. Journal für die reine und angewandte Mathematik, 606, 105-121 (2007).

[2] B. Adamczewski and Y. Bugeaud, A short proof of the transcendence of thue-morse continued fractions. American Mathematical Monthly 114, 536-540 (2007).

[3] B. Adamczewski, Y. Bugeaud, and L. Davison, Continued fractions and transcendental numbers. Ann. Inst. Fourier 56, 2093-2113 (2006).

[4] A. Baker, Continued fractions of transcendental numbers. Mathematika 9, 1-8 (1962).

[5] V. Brun, La serie 1/5 + 1/7 +... est convergente ou finie. Bull. Sci.Math. 43, 124-128 (1919).

[6] Y. Bugeaud and P. Hubert Transcendence with Rosen continued fractions J. Eur. Math. Soc. (JEMS) 15, 39-51 (2013).

[7] H. Davenport and K. F. Roth, Rational approximations to algebraic numbers. Mathematika 2, 160-167 (1955).

[8] S. Finch, Mathematical Constants. Cambridge University Press 2003.

[9] G. H. Hardy and E. M. Wright, An Introduction to the Theory of Numbers. Oxford Science Publications 1980.

[10] J. Havil, Gamma: Exploring Euler's Constant. Princeton University Press, Princeton 2003.

[11] J. Kaczorowski, The boundary values of generalized Dirichlet series and a problem of Chebyshev. Asterisque 209, 227-235 (1992).

[12] A. Y. Khinchin, Zur metrischen Kettenbruchtheorie. Compositio Mathematica 3, 275-286 (1936).

[13] A. Y. Khinchin, Continued Fractions. Dover Publications, New York 1997.

[14] P. Lévy, Sur le développement en fraction continue d'un nombre choisi au hasard. Compositio Mathematica 3, 286-303 (1936).

[15] E. Maillet, Introduction á la théorie des nombres transcendants et des propriétés arithmétiques des fonctions. GauthierVillars, Paris 1906.

[16] T. Nicely, Enumeration to $1.6 \times 10^{15}$ of the twin primes and Brun's constant. http://www.trnicely.net/twins/twins $2 . h t m l$.

[17] M. Queffélec, Transcendance des fractions continues de ThueMorse. Journal of Number Theor 73, 201-211 (1998).

[18] P. Ribenboim, The Little Book of Big Primes. 2ed., Springer 2004.

[19] M. Rubinstein and P. Sarnak, Chebyshevs bias. Experimental Mathematics 3, 173-197 (1994).

[20] C. Ryll-Nardzewski, On the ergodic theorems II (Ergodic theory of continued fractions). Studia Mathematica 12, 74-79 (1951).

[21] M. R. Schroeder, Number Theory In Science And Communication, With Applications In Cryptography, Physics, Digital Information, Computing, And Self-Similarity. Springer-Verlag New York 2006.

[22] P. Sebah, Nmbrthry@listserv.nodak.edu mailing list, post dated 22 Aug 2002. see also http://numbers.computation.free.fr/ Constants/Primes/twin.pdf.

[23] J. Sondow, Irrationality Measures, Irrationality Bases, and a Theorem of Jarnik. http://arxiv.org/abs/math.NT/0406300 2004.

[24] The PARI Group, Bordeaux, PARI/GP, version 2.3.2, 2008. available from pari.math.u-bordeaux. fr/.

[25] A. van der Poorten and J. Shallit, A specialised continued fraction. Canad. J. Math. 45(5), 1067-1079 (1993).

[26] S. S. Wagstaff Jr, Divisors of Mersenne numbers. Mathematics of Computation 40(161), 385-397 (1983).

[27] M. Wolf, Remark on the irrationality of the Brun's constant. ArXiv: math.NT/1002.41742010. 


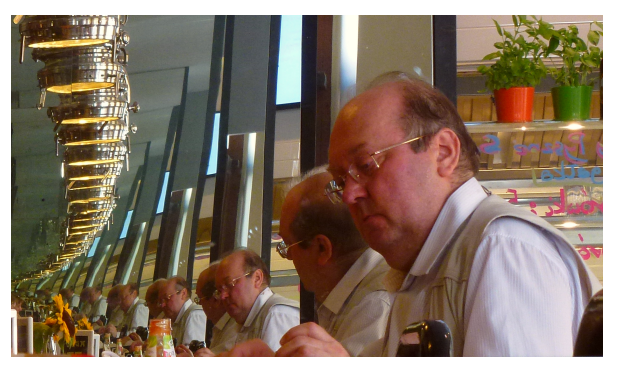

Marek Wolf, obtained PhD degree in physics (1982) and habilitation (1993) at the Wroclaw University, where he was employed till 2011. Since 1984 he has been doing computer experiments in physics and mathematics. In 1991 and 1993 he was a research fellow at the Center for Polymer Studies at the Boston University. Since 2011 he is a member of the Faculty of Mathematics and Natural Sciences, College of Sciences at the Cardinal Stefan Wyszynski University in Warsaw. His hobby is photography. 